\title{
Analyse de la flore de la forêt classée de Sanaimbo à Bongouanou - Dimbokro (Côte d'Ivoire)
}

\author{
Justin N'Dja KASSI $^{1^{*}}$, Roland Hervé KOUASSI ${ }^{2}$ et Diane Olga YONGO ${ }^{3}$ \\ ${ }^{1}$ Laboratoire de Botanique, Université de Cocody-Abidjan, 22 BP 582 Abidjan, Côte d'Ivoire. \\ ${ }^{2}$ L'Ecole Normale Supérieure d'Abidjan (ENS), 22 BP 582 Abidjan, Côte d'Ivoire. \\ ${ }^{3}$ Laboratoire de Botanique, Université de Bangui, Centrafrique. \\ *Auteur correspondant; E-mail: kassindja@yahoo.fr; Tel:00 (225) 07218492 / 00 (225) 01215462 ; \\ Fax: + $225(0) 22444473$
}

\section{RESUME}

Les investigations de terrain réalisées dans la forêt classée de Sanaimbo (Côte d'Ivoire) ont permis d'inventorier 670 espèces, qui se repartissent en 422 genres et 96 familles. Parmi ces espèces, 507 (72,5\%) sont des phanérophytes et $56(8,4 \%)$ des hémicryptophytes. 170 espèces $(25,4 \%)$ sont des lianes. La plupart des espèces possèdent de petites diaspores charnues dont la dissémination est assurée par les animaux, soit par endozoochorie ( 318 espèces $=47,5 \%)$, soit par ectozoochorie (93 espèces $=15 \%)$. Une part non négligeable des espèces $(218=32 \%)$ possède des diaspores pourvues d'appendices permettant leur dissémination par le vent. L'analyse des phytochories montre une contribution équilibrée des espèces endémiques, des espèces de liaison et des espèces à large répartition. 46 espèces (7\%) sont des guinéo-congolaises endémiques de l'Afrique de l'Ouest, dont une seule est endémique de Côte d'Ivoire. Ces différentes proportions confirment l'appartenance de la forêt étudiée à la zone de transition guinéo-congolaise/soudanienne qui correspond au secteur mésophile.

(C) 2012 International Formulae Group. All rights reserved.

Mots clés: Flore, forêt dense semi-décidue, phytogéographie, Bongouanou - Dimbokro, Côte d’Ivoire.

\section{INTRODUCTION}

La flore ivoirienne est bien connue. Des échantillons de chacune des espèces inventoriées, principalement par MM. Mangenot, Miège, Hallé et Aké Assi sont déposés à l'Herbier du Centre National de Floristique (CNF) de l'Université de CocodyAbidjan et peuvent y être consultés. De précieuses flores ont été réalisées, mais elles ne concernent que quelques groupes taxonomiques (Aké Assi, 1984 ; Kassi, 2006) sous forme d'études systématiques ou monographiques (Aké Assi, 2001-2002).
Cependant, si les forêts sempervirentes et particulièrement le Parc National de Taï (réserve de biosphère de l'UNESCO) et ses alentours ont depuis longtemps attiré l'intention des chercheurs, les forêts semidécidues restent, quant à elles, particulièrement mal connues et peu prospectées. Localement, la forêt classée de Sanaimbo n'a jamais fait l'objet d'étude ou de prospection floristique, Aké Assi (1984) ne l'a pas visitée. A cela, il convient de signaler les pressions, surtout humaines (pratiques 
agricoles entre 1972 et 1997 dans la forêt classée de Sanaimbo), qui ont sans aucun doute des effets sur la flore.

L'objectif de cette étude est de contribuer de manière significative à une meilleure connaissance de la flore de la forêt classée de Sanaimbo et, au-delà, à apporter des précisions sur la répartition des espèces à l'échelle de la Côte d'Ivoire.

\section{MATERIEL ET METHODES}

\section{Aire d'étude et Méthode}

La forêt classée de Sanaimbo est située au Centre-Est de la Côte d'Ivoire ( $6^{\circ} 20^{\prime}$ - 6²6’ N, 4³3'- 4³7 O; altitude moyenne : $120 \mathrm{~m}$ ) couvre 4322 ha sur un socle schistogranitique sur lequel se sont développés des sols ferrallitiques et hydromorphes. Le climat (moyennes annuelles : $27^{\circ} \mathrm{C}$ et $1142 \mathrm{~mm}$ ), de type attiéen avec 2 saisons sèches, détermine une végétation potentielle de type forêt dense semi-décidue à Celtis spp. et Triplochiton scleroxylon K. Schum., avec quelques petites savanes incluses. Comme la majorité des forêts du pays, elle a subi une très forte pression humaine, via la déforestation suivie d'une conversion des clairières en cultures intensives de caféiers et de cacaoyers, qui ont atteint leur plus forte extension entre 1970 et 1990 (Kassi, 2006). Aujourd'hui, les terres jadis cultivées sont, pour la plupart, en jachère et recolonisées spontanément par la forêt. La forêt classée de Sanaimbo consiste donc en un ensemble de parcelles de forêts secondaires d'âge varié (en fonction de l'ancienneté des cultures), inclus dans une matrice de forêt considérée comme primaire. Une savane arborée est également enclavée dans cette matrice.

Le plan d'échantillonnage a été conçu de manière à parcourir des reliquats de forêt primaire non exploitée, des forêts villageoises uniquement exploitées pour leur bois d'œuvre, c'est-à-dire des zones pour lesquelles la continuité forestière est assurée, mais dont la dynamique a été modifiée par l'homme et des jachères de différents âges, qui correspondent à des stades plus ou moins matures de la succession secondaire post-culturale. Des inventaires itinérants dans toutes les parties de la forêt y compris les savanes et cultures ont permis d'établir la liste floristique de cette forêt. Toutes les espèces ont été récoltées au moins une fois pour confirmer leur identification botanique à l'Herbarium de l'Université de Cocody-Abidjan, avec l'aide du Professeur Laurent Aké Assi. La nomenclature que nous avons adoptée est celle de Lebrun et Stork (1991-1997).

\section{Analyses de données \\ Nous avons établi un catalogue floristique reprenant toutes les espèces répertoriées dans cette forêt. Les types de distribution phytogéographique ont été établis suivant les grandes subdivisions chorologiques pour l'Afrique proposées par White (1986). Les types biologiques utilisés tiennent compte des modifications et ajustements proposés pour les milieux tropicaux. Concernant les modes de dissémination des diaspores en zone tropicale (graines, fruits ou toute autre partie de plante servant à la dissémination de l'espèce), nous avons suivi la classification utilisée par Sonké (1998).}

\section{RESULTATS \\ Composition floristique}

La flore locale compte 670 espèces, réparties en 422 genres et 96 familles (Tableau 1), selon APG II (2003). Deux cent dix sept espèces ont été inventoriées uniquement dans les savanes. De nombreuses espèces forestières (Trichilia prieureana A. Juss., Olax subscorpioidea Oliv, Montandra guineensis (Thonn.) A. DC., Antiaris africana Lesch. var. africana Engl., Ceiba pentandra (L.) Gaertn., Milicia excelsa (Welw.) Berg., etc.) sont présentes dans les galeries forestières des savanes. Cinquante sept autres espèces (e.g. Balanites wilsoniana Dawe et Sprague, Ongokea gore (Hua) Pierre, Leea guineensis G. Don, Placodiscus boya Aubrév. et Pellegr., Synsepalum brevipes (Baker) Pennington, etc.) ont été inventoriées uniquement en forêt. Les espèces dominantes de la voûte forestière sont, par ordre 
d'abondance décroissante : Ceiba pentandra, Triplochiton scleroxylon K. Schum., Mansonia altissima (A. Chev.) A. Chev. var. altissima, Nesogordonia papaverifera (A. Chev.) Cap., Celtis zenkeri Engl., Antiaris africana, etc. Parmi les espèces du sous-bois, on distingue de jeunes arbres de la voûte en pleine croissance, des arbustes (Trichilia prieureana, Olax subscorpioidea, etc.), des lianes (Montandra guineensis (Thonn.) A. DC., Griffonia simplicifolia (Vahl ex DC.) Baillon, Calycobolus africanus (G. Don) Heine, etc.) et des herbacées (Olyra latifolia L., Streptogyna crinita P. Beauv., Sanseviera liberica Gérôme \& Labroy), généralement en touffe. Les familles les mieux représentées, c'est-à-dire avec au moins vingt espèces sont reportées dans le Tableau 1. Ce sont des familles caractéristiques d'une flore de forêt dense humide sempervirente, à l'exception des Poaceae très présentes en savanes.

La flore de la forêt classée est constituée à $64 \%$ d'espèces endémiques et à $36,12 \%$ à distribution plus ou moins large (Figure 1). Parmi les endémiques, ce sont les guinéo-congolaises (GC) qui sont les plus représentés avec 38,65\% (259 espèces) suivies des espèces de liaison (GC-SZ, 92 espèces, soit $14 \%$ ) puis des endémiques ouestafricaine (GCW, 47 espèces, soit 7\%). Les espèces soudaniennes $\mathrm{y}$ atteignent 4,5\% (30 espèces). Les différentes proportions des espèces à large répartition sont reportées sur la Figure 1.

Le spectre biologique des espèces inventoriées (Figure 2) met en évidence une nette dominance des phanérophytes soit $72,53 \%$ (507 espèces) du total des espèces. Viennent ensuite les hémicryptophytes avec $8,37 \%$. Les géophytes, les thérophytes, les chaméphytes et les épiphytes présentent respectivement des pourcentages de 5,23\%, $5,1 \%, 4,48 \%$ et $1 \%$ de l'effectif total. Parmi les phanérophytes, $4,2 \%$ sont des individus de première grandeur (hauteur supérieure à 30 $\mathrm{m})$, mais ce sont les microphanérophytes qui dominent avec $41,26 \%$. Les lianes ligneuses représentent $25,4 \%$ de l'effectif total.

\section{Dissémination des diaspores}

Avec 47,5\% de l'effectif total viennent en tête les espèces endozochores, suivies des espèces anémochores avec $32,54 \%$.

Tableau 1: Les cinquante familles botaniques les mieux représentées en forêt classée de Sanaimbo.

\begin{tabular}{cccccc}
\hline$N^{\circ}$ & Familles & \multicolumn{2}{c}{ Genres } & \multicolumn{2}{c}{ Espèces } \\
\cline { 3 - 6 } & & \multicolumn{2}{c}{ Effectifs $(\%)$} & 85 & Effectifs $(\boldsymbol{\%})$ \\
\hline 1 & Fabaceae & 56 & 13,21 & 59 & 8,81 \\
2 & Rubiaceae & 34 & 8,02 & 44 & 6,57 \\
3 & Poaceae & 27 & 6,37 & 33 & 4,93 \\
4 & Apocynaceae & 23 & 5,43 & 30 & 4,48 \\
6 & Malvaceae & 20 & 4,71 & 27 & 4,03 \\
5 & Euphorbiaceae & 20 & 4,72 & 20 & 2,99 \\
7 & Annonaceae & 13 & 3,10 & 15 & 2,24 \\
8 & Sapindaceae & 10 & 2,36 & 21 & 3,13 \\
9 & Celastraceae & 9 & 2,12 & 19 & 2,84 \\
10 & Cyperaceae & 8 & 1,91 & 10 & 1,49 \\
11 & Asteraceae & 8 & 1,91 & 10 & 1,49 \\
12 & Menispermaceae & 8 & 1,91 & 8 & 1,19 \\
13 & Acanthaceae & 7 & 1,65 & 21 & 3,13 \\
14 & Moraceae & 6 & 1,42 & 7 & 1,05 \\
15 & Commelinaceae & 6 & 1,42 & 7 & 1,05 \\
16 & Anacardiaceae & 6 & 1,42 & 10 & 1,49 \\
17 & Verbenaceae & 5 & 1,18 & &
\end{tabular}




\begin{tabular}{|c|c|c|c|c|c|}
\hline 18 & Connaraceae & 5 & 1,18 & 9 & 1,34 \\
\hline 19 & Meliaceae & 5 & 1,18 & 8 & 1,19 \\
\hline 20 & Orchidaceae & 5 & 1,18 & 8 & 1,19 \\
\hline 21 & Amaranthaceae & 5 & 1,18 & 6 & 0,90 \\
\hline 22 & Olacaceae & 5 & 1,18 & 6 & 0,90 \\
\hline 23 & Salicaceae & 5 & 1,18 & 5 & 0,75 \\
\hline 24 & Capparidaceae & 4 & 0,94 & 6 & 0,90 \\
\hline 25 & Rutaceae & 4 & 0,94 & 6 & 0,90 \\
\hline 26 & Ulmaceae & 4 & 0,94 & 6 & 0,90 \\
\hline 27 & Araceae & 4 & 0,94 & 5 & 0,75 \\
\hline 28 & Bignoniaceae & 4 & 0,94 & 5 & 0,75 \\
\hline 29 & Ochnaceae & 4 & 0,94 & 5 & 0,75 \\
\hline 30 & Sapotaceae & 4 & 0,94 & 5 & 0,75 \\
\hline 31 & Marantaceae & 4 & 0,94 & 4 & 0,60 \\
\hline 32 & Zingiberaceae & 4 & 0,94 & 4 & 0,60 \\
\hline 33 & Convolvulaceae & 3 & 0,71 & 9 & 1,34 \\
\hline 34 & Vitaceae & 3 & 0,71 & 7 & 1,0 \\
\hline 35 & Adiantaceae & 3 & 0,71 & 6 & 0,90 \\
\hline 36 & Cucurbitaceae & 3 & 0,71 & 6 & 0,90 \\
\hline 37 & Solanaceae & 3 & 0,71 & 5 & 0,75 \\
\hline 38 & Boraginaceae & 3 & 0,71 & 4 & 0,60 \\
\hline 39 & Lamiaceae & 3 & 0,71 & 4 & 0,60 \\
\hline 40 & Rhamnaceae & 3 & 0,71 & 4 & 0,60 \\
\hline 41 & Urticaceae & 3 & 0,71 & 4 & 0,60 \\
\hline 42 & Melastomataceae & 3 & 0,71 & 3 & 0,45 \\
\hline 43 & Combretaceae & 2 & 0,47 & 11 & 1,64 \\
\hline 44 & Loganiaceae & 2 & 0,47 & 6 & 0,90 \\
\hline 45 & Ruscaceae & 2 & 0,47 & 6 & 0,90 \\
\hline 46 & Dichapetalaceae & 2 & 0,47 & 4 & 0,60 \\
\hline 47 & Passifloraceae & 2 & 0,47 & 4 & 0,60 \\
\hline 48 & Amaryllidaceae & 2 & 0,47 & 3 & 0,45 \\
\hline 49 & Arecaceae & 2 & 0,47 & 2 & 0,30 \\
\hline 50 & Hypericaceae & 2 & 0,47 & 2 & 0,30 \\
\hline
\end{tabular}

Tableau 2: Diversité spécifique et générique de quelques familles de forêts afrotropicales.

\begin{tabular}{ccccccccc}
\hline & $\begin{array}{c}\text { Sanaimbo présente } \\
\text { étude } \\
\text { (Côte d'Ivoire) }\end{array}$ & $\begin{array}{c}\text { Haute Dodo } \\
\text { (Côte d'Ivoire) } \\
\text { Kouassi (2000) }\end{array}$ & $\begin{array}{c}\text { Côte d'Ivoire } \\
\text { Aké Assi (1984) }\end{array}$ & $\begin{array}{c}\text { Forêt de N'Gotto } \\
\text { (Centrafrique) } \\
\text { Yongo (2002) }\end{array}$ \\
\cline { 2 - 8 } & genres & espèces & genres & espèces & genres & espèces & genres & espèces \\
\hline Fabaceae & 56 & 85 & 35 & 48 & 120 & 387 & 26 & 36 \\
Rubiaceae & 34 & 59 & 35 & 68 & 72 & 306 & 29 & 38 \\
Poaceae & 27 & 44 & 5 & 5 & 39 & 304 & 6 & 6 \\
Apocynaceae & 23 & 33 & 12 & 20 & 29 & 74 & 11 & 16 \\
Malvaceae & 20 & 30 & 28 & 40 & 34 & 102 & 15 & 24 \\
Euphorbiaceae & 16 & 21 & 20 & 33 & 49 & 150 & 25 & 42 \\
\hline
\end{tabular}


Tableau 3: Spectre biologique de quelques forêts denses semi-décidues d'Afrique.

\begin{tabular}{lllllll}
\hline & Ph & Hc & G & Th & Ch & Ept \\
\hline Forêt de Sanaimbo (Côte d'Ivoire) & $72,53 \%$ & $8,37 \%$ & $5,23 \%$ & $5,1 \%$ & $4,48 \%$ & $1 \%$ \\
$\begin{array}{l}\text { Forêt de Pobé au Bénin } \\
\text { (Mondjannagni, 1969) }\end{array}$ & $84 \%$ & $6,5 \%$ & $4 \%$ & $0 \%$ & $4,5 \%$ & $1 \%$ \\
$\begin{array}{l}\text { Forêt de N'Gotto (Yongo, 2002) } \\
\text { Ph : phanérophytes, Ch : chaméphytes, G : géophytes, Hc, hémicryptophytes, Th : thérophytes, Ept : épiphytes. }\end{array}$
\end{tabular}

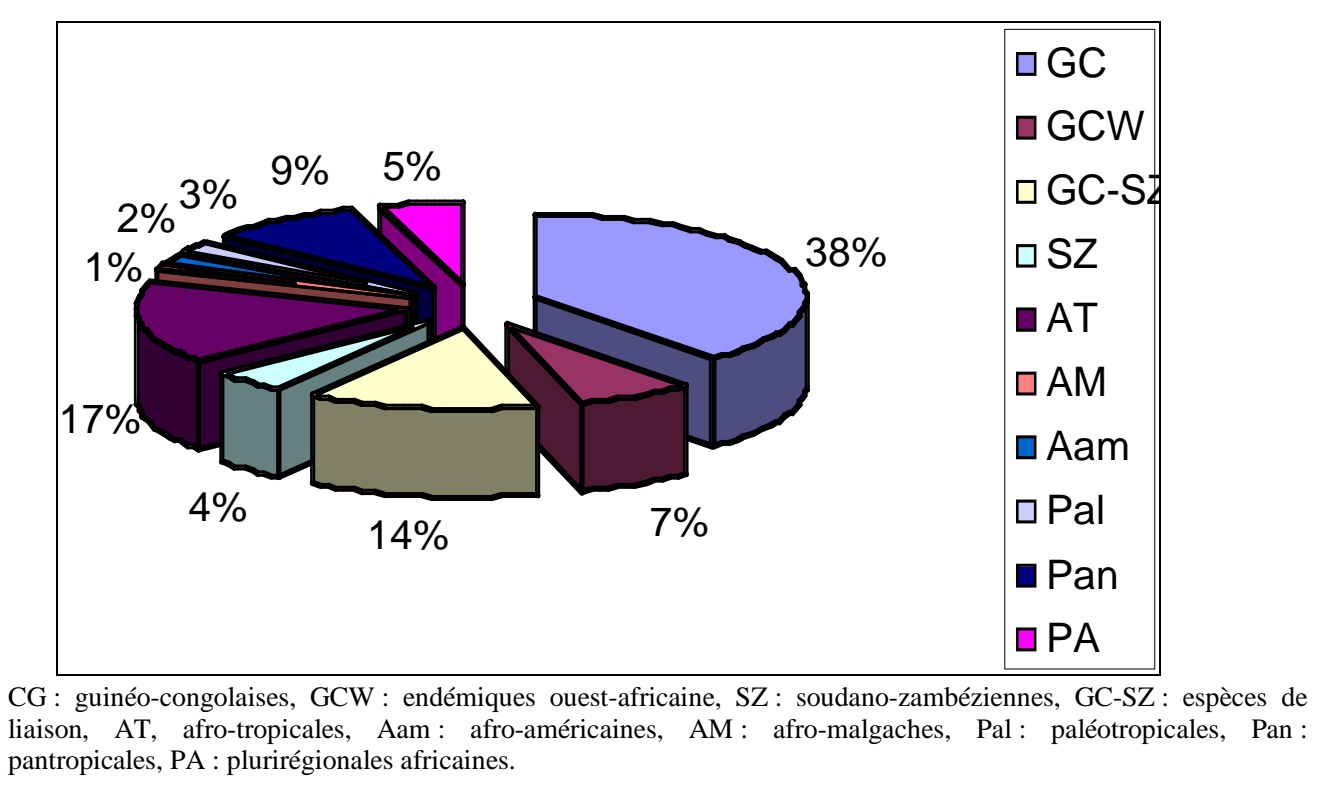

Figure 1: Spectre des phytochories de la flore de Sanaimbo.

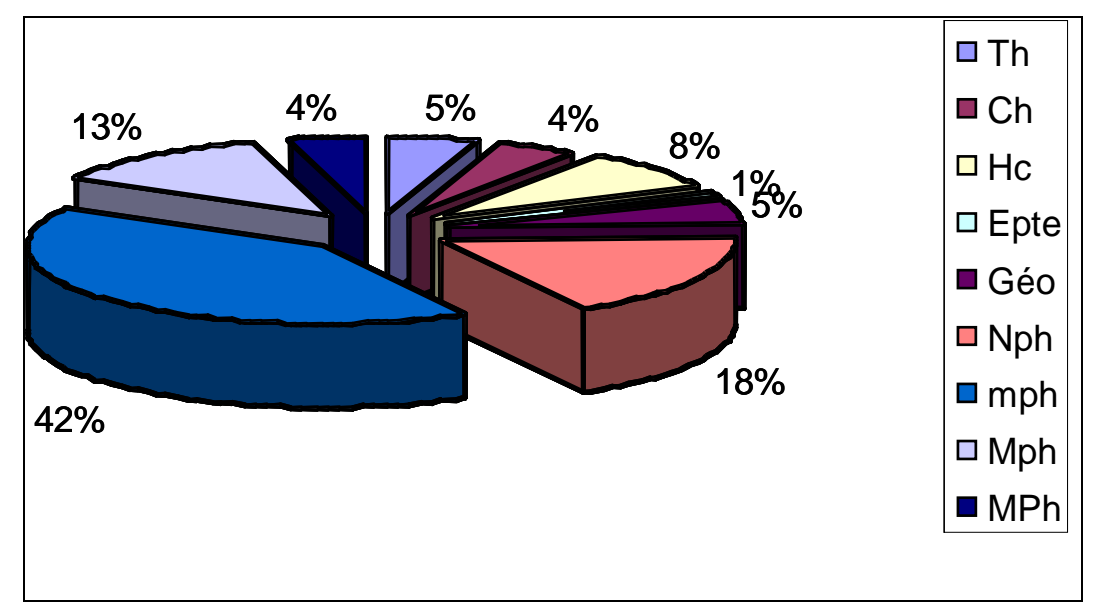

$\mathrm{Ph}$ : phanérophytes, Nph: nanophanérophytes, $\mathrm{mph}:$ microphanérophytes, Mph: mésophanérophytes, MPh : megaphanérophytes, Ch : chaméphytes, G : géophytes, Hc, hémicryptophytes, Th : thérophytes, Ept : épiphytes.

Figure 2: Spectre des types biologiques de la flore de Sanaimbo. 


\section{DISCUSSION}

Les analyses phytogéographie permettent de connaître avec précision la distribution géographique d'une ou de plusieurs espèces ainsi que l'importance des divers facteurs qui la contrôlent (Kouao, 2008). En effet, les cartes de répartition spatiale indiquent les zones de fortes diversités spécifiques appelées hotspots, la diversité spatiale des espèces et les zones d'endémisme. Elles constituent donc un outil principal pour la gestion et la conservation des espèces (Kouao, 2008; Kouao et al., 2008a). Elles permettent également de poser des hypothèses solides sur l'âge, l'origine géographique, la vitesse d'évolution et les voies de migration des taxons. Il est même possible selon Kouao, 2008, à partir de la distribution des espèces de mettre en évidence les différentes aires végétales et le découpage de la planète en unités phytogéographiques (régions, districts, secteurs, etc.).

La forêt classée de Sanaimbo, ne disposait pas de flore de référence. Nos inventaires botaniques ont permis de dénombrer 670 espèces sur une surface de 4322 hectares. Nos résultats permettent d'établir une première ébauche de catalogue floristique, valable non seulement pour cette forêt mais aussi pour toute la zone de transition forêt-savane. Les stades pionniers des jachères sont dominés par une espèce invasive : Chromolaena odorata (L.) R. King \& H. Robinson, auquel se mêle progressivement d'autres espèces pionnières : Ficus spp., Albizia spp., Rauvolfia vomitoria Afzel., Trema orientalis (L.) Blume, etc. qui sont à leur tour remplacées par des espèces cicatricielles: Trichilia prieureana, Olax subscorpioidea, Teclea verdoorniana Exell \& Mendonça, etc. La forêt se présente comme une mosaïque de peuplements à différents stades de maturité.

Elle compte 16 familles (sur les 95 , soit $15,79 \%$ ) dont l'effectif excède ou égale 10 espèces ; 31 (32,29\%) autres familles n'étant représentées que par une seule espèce. La richesse spécifique de la forêt est cependant moindre par rapport au Sud-Ouest ivoirien. Néanmoins, nos résultats sont comparables à ceux obtenus dans d'autres forêts classées de la Côte d'Ivoire. Au Sud du Parc National de Taï (100000 ha), 1233 espèces ont été inventoriées dans la forêt dense sempervirente (Dengueadhé et al., 2006). Dans la forêt de Banco (3 000 ha), bien connue sur le plan botanique, 624 espèces ont été déterminées par De Koning (1983). Rappelons que la flore de la Côte d'Ivoire comporte 3660 espèces réparties en 1218 genres et 192 familles (Aké Assi, 2001-2002). Ces résultats montrent que la forêt de Sanaimbo est aussi diversifiée que les autres forêts ivoiriennes. Elle est dominée par les familles des Fabacées sensu lato et des Rubiacées, les Poacées occupant une place non négligeable. La dominance des Légumineuses a souvent été citée comme le trait botanique caractéristique des forêts ivoiriennes (Aubréville, 1959; Guillaumet et Adjanohoun, 1971), bien que les forêts sempervirentes soient plutôt dominées par les Rubiacées (Aké Assi, 1984), comme les forêts de la région Guinéo-Congolaise en général (Sonké, 1998 ; Yongo, 2002). La richesse en Fabaceae, en Rubiaceae, en Euphorbiaceae (Tableau 2) et la présence de familles paléotropicales comme les Irvingiaceae distinguent selon Yangakola et al. (2004) ces forêts de leurs homologues néotropicales, plus riches en Lecythidaceae, Caryocaraceae, etc., alors que les forêts asiatiques sont bien connues pour leur richesse en Dipterocarpaceae.

La détermination des aires de distribution des espèces est impérative en systématique et en phytosociologie. D'abord au niveau systématique, la détermination des plantes est fondée aussi bien sur les caractères des racines, des tiges, des feuilles, des fleurs et des fruits (Kouao et al., 2008a) que sur son aire de distribution. Ensuite sur le plan floristique et autoécologique, on admet que l'aire d'une espèce est un caractère de cette espèce au même titre que ses caractères morphologiques. Enfin au point de vue 
synécologique, les facteurs spatio-temporels comptent parmi les bases de l'individualisation des communautés végétales (Habiyaremye, 1997).

Dans la forêt classée de Sanaimbo, le pourcentage des genres représentés par une espèce est très élevé. Il est égal à $69,25 \%$. Il est du même ordre de grandeur que ceux observés dans quelques forêts d'Afrique Centrale: $68 \%$ dans la réserve de faune de Dja au Cameroun (Sonké, 1998).

Le genre Diospyros est représenté par 7 espèces. Il arrive en tête dans les forêts de N'Gotto (7 espèces ; Yongo, 2002) et d'Odzada (8 espèces; Kouka, 2000). Une richesse en Diospyros est souvent considérée comme un indicateur de vieille forêt. Le spectre biologique de la forêt classée de Sanaimbo se caractérise par la dominance des phanérophytes $(72,53 \%)$. Les autres formes biologiques sont peu représentées dans la forêt. De nombreuses analyses de spectres biologiques de forêts semi-décidues aboutissent aux mêmes proportions (Tableau 3).

En moyenne, selon Trochain (1980), les arbres et les arbrisseaux représentent 52\% des espèces et les lianes ligneuses $32 \%$. Selon cet auteur, les spectres biologiques peuvent présenter certaines différences selon les régions ou les types de végétations inventoriés, mais les hémicryptophytes, les géophytes et les thérophytes sont toujours faiblement représentés, parfois même absents. Le rôle des lianes dans les milieux forestiers tropicaux en régénération a fait l'objet de nombreux travaux (Schnitzer et Bongers, 2002).

L'importance de la zoochorie est signalée dans la plupart des travaux portant sur les forêts de Côte d'Ivoire (Chapman, 1995), tout comme la dominance des espèces zoochores dans le bassin du Congo (Sonké, 1998 ; Kouka, 2000). Dans la forêt classée de Sanaimbo, les modes de dissémination des fruits et graines sont dans l'ensemble favorisés par des animaux. Cette dissémination implique surtout les oiseaux et quelques vertébrés frugivores, notamment les antilopes, les singes, les rats et les écureuils. Dans la forêt du Dja au Cameroun, Sonké (1998) a montré que beaucoup d'animaux dépendaient des espèces à fruits charnus pour leur alimentation. A l'inverse, la régénération et le maintien de ces espèces ne peuvent être assurés que si les diaspores ont été dispersées par les animaux. L'existence d'un bon équilibre entre ces deux facteurs constitue donc un gage pour la durabilité de cette forêt. Il apparaît dans nos résultats que deux modes de dissémination prédominent dans la forêt classée, en l'occurrence la zoochorie et l'anémochorie. En effet, les espèces anémochores ont une contribution non négligeable. L'importance des anémochores dans la forêt classée est due surtout à l'abondance des jachères (723 hectares soit $16,7 \%)$. Ces formations secondaires sont connues pour leur richesse en des espèces anémochores et de nombreuses lianes également anémochores. Le rôle du vent dans la dispersion des diaspores a été mis en évidence en Centrafrique dans la forêt de Ngotto (16,6\%) par Yongo (2002). En revanche, les modes barochore et hydrochore sont faiblement représentés dans ces deux forêts.

Les espèces à large distribution phytogéographique et les espèces des savanes soudaniennes sont peu nombreuses. Les espèces afro-tropicales limitées à la zone intertropicale africaine représentent $17 \%$ du spectre, avec les espèces Anthocleista djalonensis A. Chev., Crossopteryx febrifuga (G. Don) Benth. La flore de Sanaimbo et, d'une façon générale, la flore ivoirienne doivent être alors analysées dans un contexte de flore afro-tropicale. Les espèces guinéennes sont largement dominantes. Selon Sonké (1998), la forte proportion des espèces guinéennes dans le fond floristique d'une zone, est une preuve que cette zone appartient bien à la région guinéo-congolaise de White (1986). Dans la forêt classée de Sanaimbo, seulement $38,65 \%$ des espèces sont strictement confinées au centre régional d'endémisme guinéo-congolais, auxquelles s'ajoutent les endémiques ouest - africaines : 
7\%; ce pourcentage n'approche pas la prévision faite par White (1986). Pour cet auteur, la flore guinéo-congolaise est remarquablement pure avec plus de 80 à $90 \%$ d'endémiques et seulement environ $10 \%$ d'espèces d'éléments de liaison. Nos résultats sont plutôt assez proches des travaux réalisés dans les zones de transition par Yongo et de Foucault (2001).

Néanmoins, Kouao et al. (2008b) souligne que l'interprétation des aires requière $\mathrm{du}$ biogéographe une grande prudence en raison des divergences des conceptions taxonomiques et aussi de certaines distributions géographiques qui peuvent être «sujettes à caution». De plus, en pays tropicaux, les données permettant d'établir la distribution d'une espèce sont souvent incomplètes (Gioia et Pigott, 2000), soit par suite d'une absence de prospection de certains secteurs, soit en raison d'indications trop concises fournies par les observateurs ou collecteurs (Linder et al., 2005).

Selon Aké Assi (1984, 1988), la flore ivoirienne compte 62 espèces endémiques propres (ivoiriennes). Parmi ces espèces, seul Synsepalum brevipes (Baker) Pennington, est présent dans la flore de Sanaimbo. 46 autres espèces endémiques ouest-africaines, ont été inventoriées dans la forêt de Sanaimbo contre 408 espèces dans la flore ivoirienne (Crotonogyne chevalieri (Beille) Keay, Okoubaka aubrevillei Pellegr. \& Normand var. aubrevillei). L'endémisme générique de la forêt de Sanaimbo se limite à l'unique genre Okoubaka qui est un endémique Ouestafricain. Aucune des familles de la flore de Sanaimbo et de la flore ivoirienne n'est endémique de l'Ouest - africain. En prenant en compte les pressions humaines sur cette forêt classée (de 1972 à 1997), nous pouvons dire que la flore n'a pas complètement perdu sa spécificité, car la proportion des espèces à large répartition n'est que de $36 \%$, avec $14 \%$ d'espèces de liaison. $\mathrm{Au}$ contraire, ces différentes proportions confirment l'appartenance de cette zone d'étude à la zone de transition régionale guinéocongolaise/soudanienne de White (1986), qui correspond au secteur mésophile de Guillaumet et Adjanohoun (1971).

\section{Conclusion}

De cette étude floristique, il ressort que la flore de la forêt classée de Sanaimbo est très diversifiée tant au niveau des espèces, des genres que de familles. Les phanérophytes dominent, ce qui est classique dans une forêt. Néanmoins, les lianes occupent une place de choix comme dans tout milieu forestier tropical en phase de régénération. Les principaux modes de dispersion des diaspores sont la zoochorie et l'anémochorie. Les animaux joueraient un rôle assez notable dans la dissémination. Des observations sur les relations plantes-animaux permettront de préciser le rôle spécifique des animaux dans la dissémination et indirectement dans la régénération de cette forêt jadis anthropisée. La structure chorologique de la forêt classée telle qu'elle apparaît dans nos résultats est très comparable à celle des formations des zones de transition. Néanmoins, des études ultérieures sur un plus grand nombre d'espèces permettront d'expliquer davantage cette structure chorologique transitionnelle de cette forêt et par extension toute la région.

\section{REFERENCES}

Aké Assi L. 1984. Flore de la Côte d'Ivoire : étude descriptive et biogéographique, avec quelques notes ethnobotaniques. Thèse Doctorat, Université Nationale d'Abidjan, 1206 p.

Aké Assi L. 1988. Espèces rares et en voie d'extinction de la flore de la Côte d'Ivoire. Monogr. Syst. Bot. Missouri Bot. Gard., 25: 461-463.

Aké Assi L. 2001. Flore de Côte d'Ivoire Catalogue Systématique, Biogéographie et Ecologie (Tome 1). Conservatoire et Jardin Botaniques: Genève, Suisse ; 396p.

Aké Assi L. 2002. Flore de Côte d'Ivoire Catalogue Systématique, Biogéographie et Ecologie (tome 2). Conservatoire et Jardin Botaniques: Genève, Suisse ; 401p. 
APG II. 2003. An update of the Angiosperm Phylogeny Group classification for the orders and families of flowering plants. Botanical Journal of the Linnean Society, 141: 399-436.

Aubréville A. 1959. Flore Forestière de la Côte d'Ivoire (Tomes 1-3). C.T.T. Nogent sur-Marne : Paris ; 372p. ; 343p. ; 335 p.

Chapman CA. 1995. Primate seed dispersal: coevolution and conservation implication. Evolutionary Anthropology, 4: 74-82.

Dengueadhé KTS, Decocq G, Adou Yao C, Blom EC, Van Rompaey RSAR. 2006. Plant species diversity in the southern part of the Taï National Parc (Côte d'Ivoire). Biodiversity and Conservation, 15: 2123-2142.

De Foucault B. 1987. Nouvelles recherches sur les structures systématiques végétales : caractérisation, ordination, signification. Phytocoenologia, 15: 159199.

Gioia P, Pigott JP. 2000. Biodiversity assessment: A case study in predicting richness from the potential distributions of plant species in the forests of southwestern Australia. Journal of Biogeography, 27: 1065-1078.

Guillaumet J-L, Adjanohoun E. 1971. La végétation de la Côte d'Ivoire. In Le Milieu Naturel de la Côte d'Ivoire, Avenard JM, Eldin E, Girard G, Sircoulon J, Touchebeuf P, Guillaumet JL, Adjanohoun E, Perraud A (eds). Mémoires de l'ORSTOM ; 157-263.

Habiyaremye FX. 1997. Etude phytocoenologique de la dorsale orientale du Lac Kivu (Rwanda). Musee RoyaL de l'Afrique CentraLe Tervuren/Belgique. Annales des Sciences Economiques, 24: $276 \mathrm{p}$.

Kassi N'J. 2006. Successions secondaires post-culturales en forêt dense semidécidue de Sanaimbo (Côte d'Ivoire) : nature, structure et organisation fonctionnelle de la végétation. Thèse Doctorat, Université de Picardi Jules Verne, $212 \mathrm{p}$.

Kassi N'J, Decocq G. 2008. Spatio-temporal patterns of plant species and community diversity in a semi- deciduous tropical forest under shifting cultivation. Journal of Vegetation Science, 19(6): 809-820.

Koffi KJ. 2008. Analyse des structures spatiales des données de distribution phytogéographique des Acanthaceae en Afrique centrale. Thèse de Doctorat, Université Libre de Bruxelles, 225 p.

Koffi KJ, Champluvier D, Neuba FRD, Cannière C, Traoré D, Lejoly J, Robbrecht E, Bogaert J. 2008a. Analyse de la distribution des Acanthaceae en Afrique centrale: comparaison avec les théories phytogéographiques de Robyns, White et Ndjele. Sciences \& Nature, 5(2): 101-110.

Koffi KJ, Boyemba F, Ndjele M, Mate M, Robbrecht E, Lejoly J, Bogaert J. 2008b. La distribution spatiale des Acanthaceae dans les secteurs phytogéographiques de Ndjele (1988) en RD Congo. Annales de la Faculté des Sciences de Kisangani, 13: 1-8.

De Koning J. 1983. La forêt du Banco. Thèse Doctorat, Université Agronomique de Wageningen, $912 \mathrm{p}$.

Kouassi KE. 2000. Contribution à l'étude de la flore de la forêt classée de la Haute Dodo (Côte d'Ivoire). Mémoire de DEA, Université de Cocody-Abidjan, 103p.

Kouka LA. 2000. Recherches sur la flore, la structure et la dynamique des forêts du Parc national d'Odzala (CongoBrazzaville). Thèse de Doctorat, Université Libre de Bruxelles, 67p.

Lebrun JP, Stork AL. 1991-1997. Enumération des Plantes à Fleurs d'Afrique Tropicale. Conservatoire et Jardin Botanique de la Ville de Genève, Genève. Vol. 1 (249 pp.), vol. 2 (257 pp.), vol. 3 (341 pp.), vol. 4 (711 pp.).

Linder HP, Hardy CR, Rutschmann F. 2005. Taxon sampling effects in molecular clock dating: an example from the African Restionaceae. Molecular Phylogenetics Evolution, 35: 569-582.

Mondjannagni A. 1969. Contribution à l'étude des paysages végétaux du Bas-Dahomey. 
Annales de l'Université d'Abidjan G, 1: $181 \mathrm{p}$.

Schnitzer SA, Bongers F. 2002. The ecology of lianas and their role in forests. Ecology and Evolution, 17: 223-230.

Sonké B. 1998. Études floristiques et structurales des forêts de la réserve de faune du Dja (Cameroun). Thèse de Doctorat, Université Libre Bruxelles, $267 p$.

Trochain JL. 1980. Écologie végétale de la zone intertropicale non désertique. Publications Université Paul Sabatier, Toulouse, $458 \mathrm{p}$.

White F. 1986. La Végétation de l'Afrique. Recherche sur les Ressources Naturelles. ORSTOM-UNESCO: Paris XX; 384p.

Yangakola JM, de Foucault B, Yongo OD,
Lejoly J. 2004. Analyse phytogéographique comparative des savanes et des forêts de Ngotto (République Centrafricaine). Acta Botanica Gallica, 151: 124-227.

Yongo OD, de Foucault B. 2001. Analyse phytogéographique de la forêt de N'Gotto (République Centrafricaine). Systematics and Geography of Plants, 71: 843-857.

Yongo OD, 2002. Contribution aux études floristique, phytogéographique et phytosociologique de la forêt de N'Gotto (République de Centrafrique). Thèse Doctorat, Université de Lille 2 et Université de Bruxelles, 347p. 